\title{
Terapeutas Ocupacionais na Saúde Suplementar no município de Jundiaí-SP
}

\author{
Mirela de Souza Ruyza, Bruno Souza Bechara Maxta ${ }^{\mathrm{b}}$ \\ ${ }^{a}$ Terapeuta Ocupacional, Centro Universitário Padre Anchieta, Jundiaí, SP, Brasil

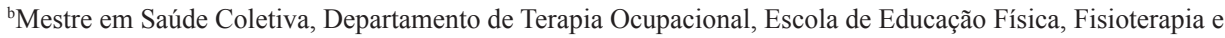 \\ Terapia Ocupacional, Universidade Federal de Minas Gerais - UMFG, Belo Horizonte, MG, Brasil
}

\begin{abstract}
Resumo: Introdução: A saúde suplementar tem se mostrado como campo emergente de atuação da Terapia Ocupacional. Objetivo: O presente trabalho objetiva reconhecer as atuais formas de participação e as propostas de atenção à saúde de terapeutas ocupacionais envolvidos na saúde suplementar no município de Jundiaí-SP. Método: Foi realizada uma pesquisa qualitativa em saúde de caráter exploratório e descritivo envolvendo a análise temática sobre a conjuntura da prática do terapeuta ocupacional. Resultados: A participação dos terapeutas ocupacionais ocorre pela vinculação do profissional em clínicas prestadoras de serviço às operadoras de saúde. A atuação dos terapeutas ocupacionais se faz em espaço ambulatorial, domiciliar e escolar, concentrada no atendimento de crianças e adultos. A abordagem de cuidado valoriza as necessidades da pessoa e a multiprofissionalidade. Conclusões: Apesar do movimento da atual Agência Nacional de Saúde Suplementar em regulamentar o setor, são desafios aos profissionais o vínculo às operadoras de planos de saúde, a apresentação de suas práticas e a sua aproximação com as atuais diretrizes de organização e cuidado da saúde suplementar no Sistema Único de Saúde.
\end{abstract}

Palavras-chave: Sistema Único de Saúde, Saúde Suplementar, Terapia Ocupacional, Prática Profissional.

\section{Occupational Therapists in the Supplemental Health care in the municipality of Jundiai, State of São Paulo}

\begin{abstract}
Introduction: Supplemental Health has become an emergent field of Occupational Therapy practice. Objectives: This study aims to identify the current forms of participation and proposals of the occupational therapists involved in the Supplemental Health care in the municipality of Jundiai, State of Sao Paulo. Method: A qualitative research on occupational therapy practice was carried out. Results: The participation of occupational therapists in the Supplemental Health care occurs in private clinics that render service to the health medical organizations - HMOs. The occupational therapists' activities are concentrated in the children and adult outpatient clinics and in the domiciliary and school spaces. Its approach values personal needs and multi-professional actions. Conclusions: Despite the actual movement of the National Agency of Supplemental Health in regulating the sector, occupational therapists face the following professional challenges: becoming contractors of the HMOs, presenting their professional practices and approaches to the Supplementary Health guidelines of the Unified Health System.
\end{abstract}

Keywords: Unified Health System, Supplemental Health, Occupational Therapy, Professional Practice. 


\section{Introdução}

A relação público-privado nos serviços de saúde fez parte das discussões sobre a saúde pública do Brasil, no início do século XX. Em 1923, em decorrência das reivindicaçóes da população urbana e industrial sobre as dificuldades de acesso aos serviços de saúde, conhecido como Lei Eloy Chaves (BRASIL, 1923) permitiu que empresas e trabalhadores contribuíssem para fundos direcionados aos seus beneficiários que ofereciam, entre outros benefícios, a assistência médica proveniente do mercado privado (BRAVO, 2006).

As assim instituídas Caixas de Aposentadoria e Pensóes (CAPs) que, mais tarde, se conformariam em Institutos de Aposentadorias e Pensões (IAPs) unificados, na década de 1960, no Instituto Nacional de Previdência Social (INPS), conformaram as estruturas pioneiras do modelo de previdência em saúde no país. Esse modelo se caracterizou pela regulaçáo do direito à saúde, considerando o trabalho formal e os setores produtivos de interesses governamentais, do mesmo modo que viabilizou o investimento do seu capital no financiamento de setores produtivos, incluindo o mercado de saúde privado. A prática médica e o cuidado especializado com a baixa eficácia às demandas gerais de saúde foram as marcas assistenciais desse modelo (ESCOREL, 2008).

$\mathrm{Na}$ década de 1980, em meio aos descontentamentos sociais e elevados custos de saúde a cada vez mais beneficiários, o modelo de saúde previdenciária torna-se torpe, sendo oportuna a sua reformulação liderada pela sociedade civil organizada, no movimento social de Reforma Sanitária Brasileira (BERTOLLI FILHO, 2008).

A redação final da Constituição de 1988 fortaleceu, em 1990, a regulamentação de um Sistema Único de Saúde (SUS) que reconheceu a saúde como direito de todos e de responsabilidade do Estado a oferta da assistência universal e integral de serviços de baixa, média e alta complexidade tecnológica a todos os brasileiros. Os serviços privados de saúde complementam o SUS através de regulação específica (BRASIL, 1990b).

Nesse processo, após a criação da Lei no 8.078, de 11 de setembro de 1990, que dispôs sobre a proteção do consumidor no país (BRASIL, 1990a), ocorreram debates sobre a formação e a atuaçáo do setor privado em conjunto com o SUS, em particular sobre a regulação dos planos de saúde no mercado brasileiro. Esses debates culminaram em uma primeira regulamentação governamental para este setor, através da aprovação da Lei no 9.656, de 3 de junho de 1998, que dispôs sobre os planos e os seguros privados de assistência à saúde monitorados pelo Departamento de Saúde Suplementar do Ministério da Saúde (BRASIL, 1998).

Esta regulamentação foi reforçada pela Medida Provisória no 2.177-44, de 24 de agosto de 2001, que definiu o Plano Privado de Assistência à Saúde como sendo a prestação continuada de serviços de assistência médica e odontológica ambulatorial e hospitalar, contratualizados por Operadora de Plano de Assistência à Saúde - representada pela pessoa jurídica que opera o serviço ou contrato (BRASIL, 2001).

Desde então, tanto as Operadoras de Plano de Assistência à Saúde (OPASs) quanto os Planos Privados de Assistência à Saúde (PPASs) são subordinadas às normas e à fiscalização da então criada Agência Nacional de Saúde Suplementar (ANS) (ALBUQUERQUE et al., 2008).

No marco legal instituído, a ANS classifica as OPASs nas seguintes modalidades. Administradora: empresas que administram exclusivamente planos de assistência à saúde; Cooperativa Médica: sociedade de pessoas sem fins lucrativos, que operam planos privados de assistência à saúde; Cooperativa Odontológica: sociedade de pessoas sem fins lucrativos que operam exclusivamente planos odontológicos; Autogestão: entidades que operam serviços de assistência à saúde ou empresas; Filantropia: entidades sem fins lucrativos que operam planos privados de assistência à saúde e tenham obtido certificado de entidade filantrópica junto ao Conselho Nacional de Assistência Social; Medicina de Grupo: empresas ou entidades que operam planos privados de assistência à saúde; Odontologia de Grupo: empresas ou entidades que operam exclusivamente planos odontológicos; e Seguradora Especializada em Saúde: sociedades seguradoras autorizadas a operar seguro (AGÊNCIA..., 2009).

Com o crescimento do número de OPASs no país, observou-se o desenvolvimento da Saúde Suplementar (SS) e a sua expansão no mercado nacional. Nesse cenário, apesar das novas oportunidades de trabalho que surgiram para os profissionais de saúde, uma série de problemas foi noticiada, decorrentes da não cobertura de procedimentos para a assistência de doenças específicas nos PPASs; sobre as diferenças contratuais e os valores cobrados entre as OPASs, ou mesmo sobre a relação de atendimento entre as OPASs e os seus clientes.

Diante da necessidade de uma maior regulação sobre o setor, a ANS aproximou-se cada vez mais das 
OPASs e de seus clientes no intuito de atualizar os registros e de promover mecanismos de transparência das informaçôes e açóes realizadas pelas OPASs. Esse movimento culminou na conquista da obrigatoriedade de assistência sobre todas as doenças reconhecidas pela Organização Mundial da Saúde nos "planos novos" registrados a partir de janeiro do ano de 1999 (BRASIL, 2001), bem como da criação do Rol de Procedimentos e Eventos em Saúde (RPES) como instrumento norteador dos serviços prestados pelas OPASs (AGÊNCIA..., 2010a).

O RPES é uma listagem dos procedimentos em saúde cujo conteúdo é revisado periodicamente. Esse instrumento considera as novas demandas e a evolução tecnológica de assistência à saúde. A sua revisão é realizada mediante consulta pública, em que usuários, prestadores de serviços, gestores do setor e as OPASs opinam sobre serviços oferecidos de forma a adaptá-los, ampliá-los ou substituí-los. Atualmente o RPES se destaca, dentre várias outras inclusôes, pelo reconhecimento do profissional terapeuta ocupacional na SS e o número de atendimentos possíveis de serem ofertados aos beneficiários de PPAS. Atualmente, é possível oferecer o número de 12 consultas/sessôes anuais para atendimentos àqueles que possuem em seus PPASs os serviços de terapia ocupacional e que atestam diagnósticos específicos (AGÊNCIA..., 2010a).

Para além da função propulsora de serviços e de fiscalização da relação de mercado com o cidadão, a ANS se empenha em orientar modelos assistenciais na SS considerando as políticas vigentes do Sistema Único de Saúde.

O modelo assistencial proposto por Malta (2003) à SS consiste na organização de redes de cuidado, envolvendo recursos físicos, tecnológicos e humanos pelas OPASs aos seus beneficiários. Esta autora critica a tradicional organização do trabalho no setor privado marcada por práticas fragmentadas e especializadas de atençáo à saúde, em fluxos que priorizam o acesso àqueles que podem arcar financeiramente com as despesas de saúde ou àqueles que detêm os PPASs com maior cobertura de serviços.

Sob esta perspectiva, torna-se imperativo compreender e qualificar o processo de cuidado na SS, investindo em abordagens assistenciais acolhedoras, em diagnósticos compartilhados com diretrizes terapêuticas reconhecidas, valendo-se da corresponsabilidade entre profissionais e serviços, independente da locação pública ou privada, sobre as açóes orientadas à necessidade ou ao problema de saúde apresentado pela pessoa. Torna-se oportuno provocar a construção de um cuidado na SS pautado sobre o profissional de referência que permita o desenvolvimento de práticas multiprofissionais e interdisciplinares de assistência entre os serviços vinculados às OPASs e com objetivos confluentes em propostas de cuidado espelhados no SUS (BRASIL, 2008, 2004).

\section{Objetivo}

Este estudo tem como objetivo reconhecer as atuais formas de participação e as propostas de atenção à saúde, de terapeutas ocupacionais, envolvidos na Saúde Suplementar no município de Jundiaí-SP.

\section{Método}

Para esta trajetória, propomos a realização de uma pesquisa qualitativa em saúde de caráter exploratório e descritivo (MINAYO, 2004) com terapeutas ocupacionais atuantes na SS no município de Jundiaí-SP.

Como estratégias de instrumentalização da pesquisa de campo, foram utilizadas fontes de informaçôes secundárias, disponíveis no Sistema de Informação do Ministério da Saúde - DATASUS, nas bases de dados da Agência Nacional de Saúde Suplementar e do Cadastro Nacional de Estabelecimentos de Saúde, entre os meses de setembro de 2009 a outubro de 2010 , no intuito de identificar o contexto da SS na região de Jundiaí-SP e os terapeutas ocupacionais atuantes nesse campo.

De maneira complementar, entrevistas semiestruturadas foram desenvolvidas com terapeutas ocupacionais que atuam na SS com vistas a dialogar sobre a formação profissional; sobre a sua entrada no setor; a atual forma de participaçáo; e a maneira como sua prática é apresentada. Dos profissionais identificados, quatro aceitaram participar da pesquisa. Cada participante recebeu um termo explicativo dos eixos e objetivos da pesquisa, bem como o consentimento de sua participação. Os encontros aconteceram em local seguro, pertinentes aos terapeutas ocupacionais e aos pesquisadores. As entrevistas tiveram tempo de duração entre uma e três horas. As falas dos participantes foram registradas em áudio e transcritas em documento de texto.

Como forma de apreciação dos dados, foi realizada a análise temática pela tabulação e a articulação discursiva entre os dados empíricos da pesquisa.

\section{Resultados e discussão}

Segundo informaçôes do Instituto Brasileiro de Geografia e Estatística, o município de Jundiaí possui uma população estimada em 350.000 habitantes (INSTITUTO..., 2010). Atuam na Saúde 
Suplementar no município aproximadamente 255 OPASs, que oferecem serviços de média e alta complexidade aos seus clientes (AGÊNCIA..., 2010b). Em junho de 2010, o município possuía uma das maiores taxas de cobertura de SS do país com 257.173 beneficiários de assistência médica privada, concentrando maior número nas faixas etárias de 25 a 29 anos (AGÊNCIA..., 2010c). Esse valor representa $70 \%$ da população municipal.

O Cadastro Nacional de Estabelecimentos de Saúde indicou a existência de 37 terapeutas ocupacionais atuantes em estabelecimentos de saúde no município de Jundiaí (BRASIL, 2010). Considerando as informaçóes gerais sobre os serviços prestados por esses estabelecimentos, foram observados que $90 \%$ desses profissionais atuavam em estabelecimentos privados e/ou filantrópicos que ofereciam serviços especializados no campo da reabilitação física $(93,75 \%)$ e da saúde mental (6,25\%) para a população.

Não foi possível identificar quais destes serviços estáo vinculados as OPASs promotoras no município.

Deste universo, as quatro profissionais participantes do estudo se apresentam como profissionais atuantes na SS. Todas são do sexo feminino e se formaram em uma mesma universidade no estado de São Paulo. As profissionais possuem formação especializada para atuar no contexto de saúde educacional (TO2), ambulatorial (TO1, TO2, TO3, TO4) e hospitalar (TO1, TO2, TO3, TO4). Três delas possuem mais de 20 anos de experiência profissional em serviços públicos de saúde. Uma das profissionais atua no contexto da SS no município desde o ano de 1995.

Essas profissionais declaram atuar na SS por intermédio de clínicas privadas prestadoras de serviços às OPASs da região no âmbito da reabilitação física nas áreas de neurologia (TO 1, TO 2), ortopedia (TO 1, TO 2, TO 3, TO 4) e estimulação sensorial (TO 2) no cuidado a crianças (TO 2) e adultos (TO 1, TO 3, TO 4).

Segundo as profissionais, as clínicas contratualizam junto às OPASs um conjunto de serviços especializados, de acordo com a sua atual equipe profissional e a sua capacidade de atendimento. Estes serviços são identificados nos PPASs sobre a forma de procedimentos. Através dos PPASs, os clientes que possuem em sua cobertura os serviços de terapia ocupacional buscam o cuidado com o profissional.

Este cuidado é iniciado com o encaminhamento médico, geralmente por ortopedistas, reumatologistas e/ou neuropediatras.
Em um segundo momento, são realizadas triagens e avaliaçóes pelo profissional utilizando-se de instrumentos padronizados e não padronizados de avaliação. Para a elaboração e a pactuação de plano de tratamento com o cliente e seus familiares, são considerados os resultados apresentados pela avaliação, os objetivos almejados e os procedimentos disponíveis nos PPASs.

Você faz uma avaliação inicial para ter um ponto de partida e depois, durante o tratamento, vai fazendo reavaliaçóes, vai constantemente reavaliando e mudando seu planejamento de tratamento. (TO2)

O paciente chega e faço uma avaliação aberta, entrevista com a familia, avaliação prévia, e no decorrer do atendimento, uma avaliação especifica e orientação para a família. (TO1)

Nos processos de terapia ocupacional, os clientes são atendidos, em média, uma ou duas vezes por semana, respeitando o projeto elaborado. Nestes, são incorporadas as práticas de visitas domiciliares e escolares para adequação ambiental e educação em saúde, quando necessária.

"Quando precisa de adaptaçóes, tem que ir na escola para explicar o trabalho e a escola apoia o tratamento." (TO2)

Sobre a prática relatada, foi evidente o empenho de todos para a construção multidisciplinar do cuidado em conjunto com fisioterapeutas, psicólogos e fonoaudiólogos. A realização deste trabalho é possível quando esses profissionais fazem parte $\mathrm{da}$ mesma clínica. Os momentos de discussóes de casos clínicos são valorizados.

"Não temos supervisão formal, mas discutimos os casos com todos da equipe (...) saber das patologias; há troca de materiais sem pedir. É dinâmico." (TO3)

"Atualmente, faço discussóes em equipe." (TO2)

Nesta perspectiva, o objetivo último do profissional enquadra-se na promoçáo da independência e da autonomia das pessoas respeitando o contexto e os recursos de vida dos seus pacientes. $\mathrm{O}$ atendimento é construído considerando o aporte multidisciplinar, particularmente quando o profissional possui ou consegue o contato do profissional que encaminhou a pessoa. Do mesmo modo, são investimentos as atuaçóes do terapeuta ocupacional em settings que não correspondem à ambiência ambulatorial das clínicas, apontando para inovaçôes dos tradicionais procedimentos de reabilitação para outros que contemplam a prevenção e a promoção de saúde. 
No entanto, essas abordagens ora são potencializadas, ora inibidas pelos vínculos de trabalho do terapeuta ocupacional com as clínicas prestadoras de serviços. Dos profissionais entrevistados, $75 \%$ são funcionários contratados das clínicas e $25 \%$ são seus proprietários.

Segundo as terapeutas ocupacionais contratadas pelas clínicas, dificuldades existem no manejo dos casos quando há a necessidade de desenvolver procedimentos que náo fazem parte do pacote de serviços disponibilizados aos clientes nos PPASs, ou quando é identificada a necessidade de desenvolver procedimentos que não foram contratualizados entre as clínicas e as OPASs.

Esses profissionais possuem pouco poder de negociação com as OPASs. Foram relatos de que os pacientes, quando esgotam o seu pacote básico de procedimentos ou não conseguem custear os demais serviços necessários ao seu tratamento, os profissionais ou assumem a realização desses procedimentos sem custos ao cliente e ônus à clínica, ou eles encaminham o paciente a algum serviço público de saúde. Caso o profissional náo consiga reorientar o processo iniciado, o atendimento é interrompido.

A maioria dos pacientes que o convênio não cobre paga particular, e aqueles que não podem, eu encaminho para o centro de reabilitação do municipio (...) assim como teve clientes que já vinham no particular e aproveitaram as seis sessóes para dar um suspiro e depois retornar ao particular. (TO2)

Se o paciente necessita de mais atendimentos, é dito para o paciente que ele sabe o que é melhor para ele; conversa; estabelece valores; ou nosso valor fechado; estuda cada caso; não deixa de atender o paciente antes de conversar com ele. Temos uma responsabilidade enquanto equipe. (TO3)

Já os terapeutas ocupacionais proprietários das clínicas expressam, para além das dificuldades em lidar com as regras de oferta e de regulação das OPASs, que a formalização de seus vínculos com as operadoras é um processo economicamente inseguro e administrativamente burocrático.

"Não acho benéfico fazer um atendimento em seis sessóes pelo plano de saúde e também porque paga muito baixo." (TO1)

Foram identificados que as OPASs fazem diversas exigências para credenciar o profissional, tais como registro de pessoa jurídica, títulos de especializaçóes acadêmicas, além de ter conta única no banco para pagamento dos procedimentos realizados. Segundo as entrevistadas, tais exigências aumentam as contribuiçôes fiscais e provocam a necessidade de investimentos constantes do profissional para a captaçáo de recursos financeiros e para o aprimoramento profissional, que atualmente não é compensado pelos lucros adquiridos nos contratos vigentes.

\begin{abstract}
Dependendo do plano, você acaba recebendo muito pouco e precisa ter um número grande de pacientes para valer a pena, para manter um consultório. Para mim não foi fácil entrar nos planos de saúde porque na época que entrei a TO não tinha reconhecimento e nem a necessidade que tem hoje. Meu credenciamento é de 1995. Eaconteceu porque as pessoas conheciam meu trabalho e indicavam meu nome para ser credenciada, hoje ficou mais fácil pela lei e pela obrigatoriedade de os planos terem o serviço. Antes só tinha o serviço porque achavam que o serviço era interessante, $e$ não porque era obrigado a ter. (TO4)
\end{abstract}

Os baixos valores pagos pelos procedimentos fizeram com que algumas terapeutas ocupacionais buscassem novas oportunidades na SS, pela oferta de projetos de prevenção a saúde, ou até mesmo através da readequação do seu processo de trabalho, investindo no atendimento a vários pacientes ao mesmo tempo através de grupos.

Tem também um projeto que a gente desenvolve com um plano de saúde, de prevenção de incapacidades com os pacientes de artrite reumatoide, osteoartrose, lombalgia, fibromialgia e osteoporose. Ele passa por um programa de quatro meses. A gente ensina todo o tratamento e a reabilitação que ele tem que fazer, e no final desses quatro meses ele tem alta. $O$ atendimento é de uma hora, de uma a duas vezes por semana. (TO4)

Diante dessas dificuldades, as profissionais reconhecem a importância do encaminhamento médico, e contam com esse procedimento como dispositivo de ampliação do número de atendimentos e reconhecimento da terapia ocupacional no setor.

O médico que encaminhou o paciente, ele já te encaminhou com o conhecimento que você vai poder ajudar o paciente dele, temos um respeito e cuidado com esse ser humano. (TO3)

Aumentou a quantidade de pacientes, porque os próprios médicos estão na maioria dos convênios. Antes eles tinham limitaçōes para mandar para a gente, agora já mandam com tranquilidade porque o convênio cobre. (TO2)

Apesar do reconhecimento positivo que esses encaminhamentos trazem para a ampliação do número de clientes e para a visibilidade da prática do terapeuta ocupacional, essa postura profissional 
estimula a conduta médica como reguladora para a prática desse profissional na SS. Sob esse bojo, a construçáo da autonomia do profissional no setor e a sua proposta de ampliar e integrar o cuidado prestado pelos diferentes profissionais tornam-se ainda mais desafiadoras, tendo em vista que a lógica das OPASs atuantes na SS neste município parece ser, justamente, a de reduzir e segmentar a assistência em saúde dividindo as demandas e procedimentos para cada núcleo profissional vinculado aos PPASs.

Para mudanças desse contexto, espaços de discussão sobre a SS são solicitados e necessários ao terapeuta ocupacional no intuito de compreender melhor o seu funcionamento, de valorizar-se enquanto profissional e direcionar açóes que visem a qualidade do cuidado prestado considerando, entre as inúmeras prerrogativas deontológicas de sua profissão, o direito à saúde $\mathrm{e} o$ respeito às demandas identificadas.

\section{Conclusões}

A participação dos terapeutas ocupacionais na SS na regiāo de Jundiaí ocorre pela vinculação do profissional em clínicas privadas prestadoras de serviço às operadoras de planos de saúde. O modelo de cuidado apresentado pelos terapeutas ocupacionais entrevistados busca a prática multiprofissional centrada na pessoa em espaço ambulatorial, domiciliar e escolar visando a prevenção, a reabilitação e a promoçâo de independência e autonomia de pacientes com o apoio das famílias.

Mesmo com avanços na construção de processos de cuidado ampliado, são desafios emergentes aos terapeutas ocupacionais: qualificar as características organizacionais da SS; atuar em rede de serviços considerando a complementaridade da SS no SUS; o exercício da prática integral e multiprofissional em saúde; o desenvolvimento de habilidades e de estratégias de negociação junto às OPASs; e o exercício de sua autonomia profissional no intuito de um trabalho cada vez mais reconhecido pela sua identidade e resultados em saúde, e menos pelos seus procedimentos oferecidos.

\section{Referências}

AGÊNCIA NACIONAL DE SAUDE SUPEMENTAR - ANS. Informaçōes em Saúde Suplementar. Rio de Janeiro: ANS, 2009. Disponível em: <http://www. ans.gov.br/anstabnet/anstabnet/notas_receita.htm>. Acesso em: 12 nov. 2009.

AGENCIA NACIONAL DE SAUDE SUPEMENTAR - ANS. Resolução Normativa $n^{\circ} 211$, de 11 de janeiro de 2010. Atualiza o Rol de Procedimentos e Eventos em Saúde. Diário Oficial da República Federativa do Brasil, Poder Executivo, Brasília,
DF, 12 jan. 2010a. Seção 1, n. 7. Disponível em: <http:// www.ans.gov.br/portal/site/legislacao/legislacao_integra. asp?id=1974\&id_original >. Acesso em: 12 abr. 2010.

AGÊNCIA NACIONAL DE SAÚDE SUPLEMENTAR - ANS. Operadoras com registro ativo. Rio de Janeiro: ANS, 2010b. Disponível em: <http://www. ans.gov.br/anstabnet/anstabnet/deftohtm.exe?anstabnet/ dados/TABNET_03A.DEF>Acesso em: 20 out. 2010.

AGÊNCIA NACIONAL DE SAÚDE SUPLEMENTAR - ANS. Beneficiários por Município. Rio de Janeiro: ANS, 2010c. Disponível em: <http://www. ans.gov.br/anstabnet/anstabnet/deftohtm.exeanstabnet/ dados/TABNET_02.DEF>Acesso em: 20 out. 2010.

ALBUQUERQUE, C. et al. A situação atual do mercado da saúde suplementar no Brasil e apontamentos para o futuro. Ciência e Saúde Coletiva, Rio de Janeiro, v. 13, n. 5, p. 1421-1430, 2008. http://dx.doi.org/10.1590/ S1413-81232008000500008

BERTOLLI FILHO, C. B. História da Saúde Pública no Brasil. São Paulo: Ática, 2008.

BRASIL. Senado Federal. Decreto no 4.682, de 24 de janeiro de 1923. Cria, em cada uma das empresas de estradas de ferro existentes no país, uma Caixa de Aposentadoria e Pensōes para os respectivos empregados. Diário Oficial da República Federativa do Brasil, Poder Executivo, Brasília, DF, 28 jan. 1923. Seção 1.

BRASIL. Senado Federal. Lei no 8.078 , de 11 de setembro de 1990. Dispóe sobre a proteçâo do consumidor e dá outras providências. Diário Oficial da República Federativa do Brasil, Poder Executivo, Brasília, DF, 12 set. 1990a. Seção 1.

BRASIL. Ministério da Saúde. Lei no 8.080, de 19 de setembro de 1990. Dispóe sobre as condiçóes para a promoção, proteção e recuperação da saúde, a organização e o funcionamento dos serviços correspondentes e dá outras providências. Diário Oficial da República Federativa do Brasil, Poder Executivo, Brasília, DF, 20 set. 1990b. Seção 1.

BRASIL. Ministério da Saúde. Lei no 9.656, de 03 de junho de 1998. Dispóe sobre a elaboração do rol de procedimentos e eventos em saúde que constituirão referência básica e fixa as diretrizes para a cobertura assistencial. Diário Oficial da República Federativa do Brasil, Poder Executivo, Brasília, DF, 4 jun. 1998. Seção 1.

BRASIL. Ministério da Saúde. Medida Provisória $\mathrm{n}^{\circ}$ 2.177-44, de 24 de agosto 2001. Altera a Lei no 9.656, de 3 de junho de 1998, que dispóe sobre os planos privados de assistência à saúde e dá outras providências. Diário Oficial da República Federativa do Brasil, Poder Executivo, Brasília, DF, 25 ago. 2001. Seção 1.

BRASIL. Ministério da Saúde. Secretaria de Atenção à Saúde. Núcleo Técnico da Política Nacional de Humanização. Secretaria-Executiva. HumanizaSUS: a clínica ampliada. Brasília: Ministério da Saúde, 2004.

BRASIL. Ministério da Saúde. Secretaria de Atenção à Saúde. Núcleo Técnico da Política Nacional de Humanização. Clínica ampliada: equipe de referência e projeto terapêutico singular. Brasília: Ministério da Saúde, 2008. 
BRASIL. Ministério da Saúde. Departamento de Informação em Saúde. Cadastro Nacional de Estabelecimentos de Estabelecimentos de Saúde. Consulta de Profissionais. Disponível em: <http://cnes.datasus.gov.br/>. Acesso em: 16 jan. 2010.

BRAVO, M. I. S. Política de Saúde no Brasil. In: MOTTA, A. E. Serviço Social e Saúde: formação e trabalho profissional. São Paulo: Cortez, 2006. p. 88-110.

ESCOREL, S. História das Políticas de Saúde no Brasil de 1964 a 1990: do golpe militar à Reforma Sanitária. In: GIOVANELLA, L. et al. Politicas e sistemas de saúde no Brasil. Rio de janeiro: FIOCRUZ, 2008. p. 385-434.
INSTITUTO BRASILEIRO DE GEOGRAFIA E ESTATISTICA - IBGE. Cidades@.Jundiai. Rio de Janeiro: IBGE, 2010. Disponível em: <http://www.ibge. gov.br/cidadesat/painel/painel.php?codmun=352590>. Acesso em: 10 jun. 2010.

MALTA, D. C. Saúde Suplementar e Modelos Assistenciais. Rio de Janeiro: ANS, 2003. p. 1-48. Disponível em: <http://www.ans.gov.br/data/files/8A958865266CAFE 201267FB88E522BBC/TT_AS_14_DCarvalhoMalta_ ModelosAssistenciais.pdf 2003>. Acesso em: 18 jun. 2010. MINAYO, M. C. S. O desafio do conhecimento: pesquisa qualitativa em saúde. São Paulo: Hucitec, 2004.

\section{Contribuição dos Autores}

Mirela de Souza Ruyz: Responsável pela concepção do artigo, revisão e organização bibliográfica, realização das entrevistas, análise e discussão dos dados, redação e revisão final do texto. Bruno Souza Bechara Maxta: Responsável pela concepção do artigo, orientação técnica e metodológica da pesquisa, análise e discussão dos dados, e revisão final do texto. 
